\title{
Complicated Sonographic View of Diffuse Hepatic Steatosis
}

\author{
MELANIA ARDELEAN ${ }^{1}$, ROXANA BUZAS ${ }^{1}, *$, DANIEL LIGHEZAN ${ }^{1}$, BASA NORINA $^{1}$, \\ CORINA DUDA-SEIMAN², DANIEL DUDA-SEIMAN ${ }^{3}$, NICOLAE CONSTANTIN BALICA ${ }^{4}$, \\ OVIDIU ARDELEAN ${ }^{5}$ \\ 1"Victor Babes" University of Medicine and Pharmacy, Internal Medicine Department, 2 Eftimie Murgu Sq., 300041, \\ Timisoara, Romania \\ ${ }^{2}$ West University, Chemistry, Biology and Geography Faculty, 4 Vasile Parvan Blvd., 300223, Timisoara, Romania \\ 3"Victor Babes" University of Medicine and Pharmacy, Department of Cardiology - Ambulatory Internal Medicine, \\ Preventive Cardiology and Cardiovascular Rehabilitation, 2 Eftimie Murgu Sq., 300041, Timisoara, Romania \\ 4"Victor Babes" University of Medicine and Pharmacy, ENT Department, 2 Eftimie Murgu Sq., 300041, Timisoara, Romania \\ 5"Victor Babes" University of Medicine and Pharmacy, Surgery Department I, 2 Eftimie Murgu Sq., 300041,Timisoara, \\ Romania
}

The aim of this study was to reveal the important role of CEUS in identifying correctly benign hepatic lesion vs a malignant one. We report a case of a diffuse hepatic steatosis particular sonographic aspect in a middle aged male presenting with general malaise. History revealed cardiac pathology, diabetes mellitus type 2 and chemotreated prostate neoplasia. The abdominal ultrasound (US) presented numerous hyperechoic lesions disseminated in the whole parenchyma. A contrast enhanced ultrasound (CEUS) of the lesions described an isoenhancement pattern of the lesions in the arterial phase, followed by the same aspect in the venous phase, with a high uptake pattern in the late phase. The CT and MRI also confirmed a diagnosis of a benign pathology. Hepatic steatosis may occur in chemotreated cancer patients, raising a suspicion of hepatic metastasis, but also due to the amount of lipid deposit distribution in the liver. Thus, CEUS was able to establish a correct diagnosis in a fast and reliable way.

Keywords: hepatic steatosis, isoenhancement, contrast enhanced ultrasound

Diffuse and focal liver fatty infiltration (hepatic steatosis) has been described in association with chemotherapy, alcohol abuse, diabetes mellitus, obesity, exogenous steroids, iv hyperalimentation [1-13]. Focal hepatic steatosis can be misinterpreted for other hepatic lesions: metastases, infiltrative primary liver neoplasms, abscesses and cysts [14].

We present a case of an unusual pattern of fatty liver infiltration, which may have been caused by insulin, past medical history of chemotreated prostatic neoplasia, revealed by a gold standard imagistic method - computed tomography or a magnetic resonance imaging (CT/ MRI).

\section{Experimental part}

A 50 years-old man was referred to Internal Medicine Department with general malaise. An abdominal sonography (US) of the liver was performed at a General Practitioner's office. The patient was referred to us with "Suspicion of metastasis". The patient, known with cardiological pathology, diabetes mellitus type 2 insulin dependent, radiochemotreated and surgically treated prostate cancer, 2 years ago, was hospitalized, and a second an abdominal liver sonography was performed: liver with multiple hyperechogenic images of different sizes disseminated over the entire surface, without other pathological changes (Fig.1).

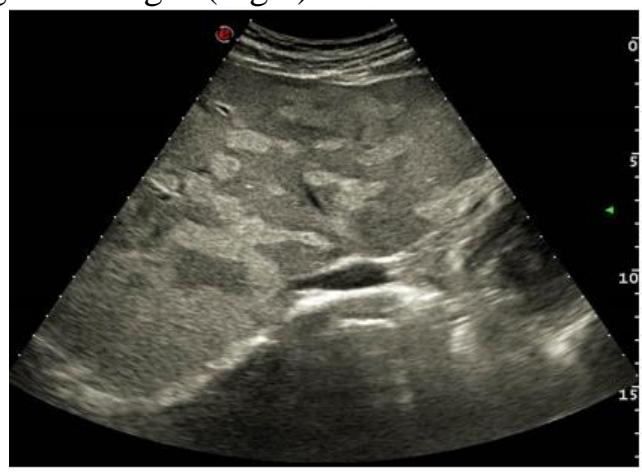

Fig.1Multiple

hyperechogenic lesions in the liver's parenchyma.

*email: roxanabuzas@yahoo.com 
A contrast enhanced ultrasound (CEUS) of the liver was performed using MyLab ClassC - Esaote - Sonovue (Bracco, Italy), with Sonovue (Sulphur hexafluoride - SF6) I.V $2.4 \mathrm{~mL}$ in one injection. The acquisition of SF6 was followed: 10 to 30 seconds arterial time, 30 to 120 seconds venous time, $>120$ seconds parenchymatous time. The observed lesions uptake the SF6 progressively started at 30 seconds, in venous time the uptake being similar with the parenchyma. High uptake was maintained over 120 seconds (parenchymatous time). A diagnosis of diffuse hepatic steatosis was given. This type of presentation of diffuse hepatic steatosis may suggest a neoplastic disease false diagnosis. Using the contrast ultrasound, we were able to confirm the diagnosis in a fast and reliable way (Fig.2).

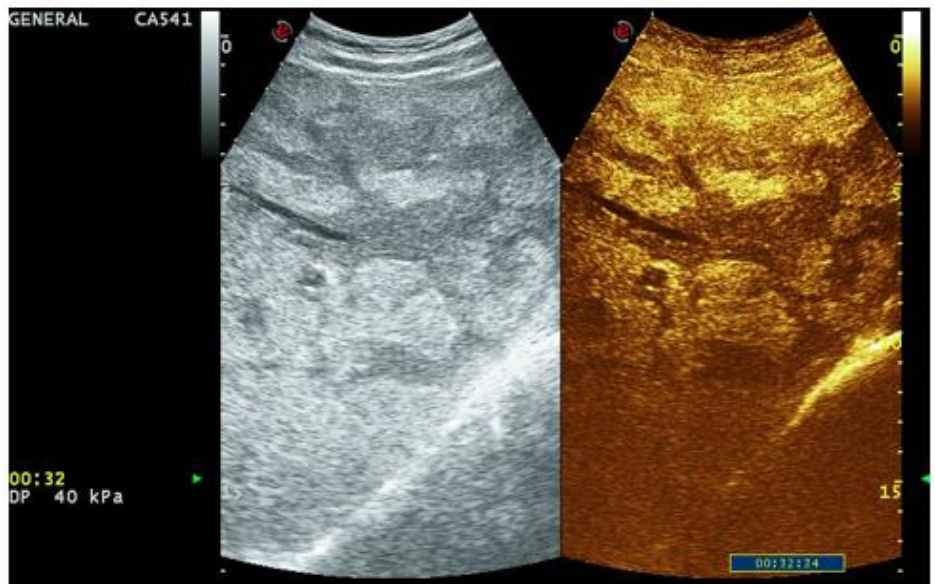

Fig. 2 Isoenhanced lesion during the arterial phase

Further, both a CT scan and a MRI with contrast were performed, confirming the diagnostic of a benign pathology.

\section{Results and discussions}

Unusual and heterogeneous forms of fatty deposition may prevent greyscale US from reliably ruling out the presence of liver metastases, in cancer patients, even in the absence of any suspicion of liver lesions [4]. Fatty change of the liver, often occurring in cancer patients, that underwent chemotherapy and presenting with different patterns of deposition, may further hamper the proper assessment by means of US [1, 6, 9]. As Rafaelsen et al [10] and Tommaso V et al [11] suggest in their studies, CEUS has proved to have an important role as a first line tool for ruling out the presence of metastases in cancer patients, inconclusive in US, but with a fatty infiltration of the liver.

According to EFSUMB (5) criteria, also the same aspect encountered in our case, the diagnostic criteria for metastatic lesions shows a hypovascular or a hyperevascular enhancement in the arterial phase, followed by a hyenhancement and wash-out the portal and late phase. The diagnostic criteria for fatty liver infiltration presents an isoenhancement pattern in all phases.

Hepatic steatosis may have variable appearance depending on the amount of lipid deposited, the distribution of the lipids in the liver and the presence of associated hepatic disease. Diffuse hepatic steatosis may have a nodular aspect, which can mimic a malignant disease.

Insulin proved to have an important place in the pathogenesis of hepatic steatosis [7, 12]. In the fasting state (low insulin) free acids in the liver are preferentially oxidated to ketone bodies. In the feeding time, high insulin levels inhibit the oxidation of free acids and sustain the esterification of free acids into triglycerides, which accumulates in the liver. Like in our study, the patient being diabetic, the pattern of hepatic steatosis might be explained by the higher insulin concentration in the liver periphery. The same pattern of hepatic steatosis was met in Wanless and Kilworth's studies $[7,12]$.

\section{Conclusions}

The detection rate for liver metastases by CEUS is reported to be similar by CT or MRI, with no significant differences, but most studies found the sensitivity of CEUS to be slightly higher $[2,8]$.

The authors declare that the study was performed in accordance with Declaration of Helsinky regarding ensure research involving human subjects, the patient has given his informed, written consent for the study.

\section{References}

1.BIPAT, S., VAN LEEUWEN, M.S., COMANS, E.F., PIJL, M.E., BOSSUYT, P.M., ZWINDERMAN, A.H., STOKER, J., Abdom Imaging 39, 2009, p.193.

2.BIPAT, S., VAN LEEUWEN, M.S., COMANS, E.F., PIJL, M.E., BOSSUYT, P.M., ZWINDERMAN, A.H., STOKER, J., Radiology 237, 2005, p.123.

3.BRAWER, M.K., AUSTIN, G.E., LEWIN, K.J., Gastroenterology 78, 1980, p. 247. 
4.DÉCARIE, P.O., LEPANTO, L., BILliARD, J.S., OLIVIÉ, D., MURPHY-LAVALLÉE, J., KAUFFMANN,C., TANG, A., Insights Imaging 2, 2011, p. 533.

5.*** European federation of Societies for Ultrasound in Medicine and Biology-EFSUMB.

6. HAMER, O.W., AGUIRRE, D.A., CASOLA, G., SIRLIN, C.B., Radiographics 26, 2006, p. 1637.

7.KILWORTH, L., CRANE, D., MASTERS, C., Biochem Int., 10, 1987, p. 539.

8.PISCAGLIA, F., CORRADI, F., MANCINI, M., GIANGREGORIO, F., TAMBERI, S., UGOLINI, G., COLA, B., BAZZOCCHI, A., RIGHINI, R., PINI, P., FORNARI, F., BOLONDI, L., BMC Cancer 7, 2007, p. 171.

9.QUAIA, E., D'ONOFRIO, M., PALUMBO, A., ROSSI, S., BRUNI, S., COVA, M., Eur Radiology 16, 2006, p. 1599.

10.RAFAELSEN, S.R., JAKOBSEN, A., Colorectal Dis., 13, 2011, p. 420.

11. BARTOlOTTA, T.V., TAIBBI, A., PICONE, D., ANASTASI, A., MIDIRI, M., LAGALLA, R., Ultrasonography 36, 2017 , p.160.

12.WANLESS, I.R., BARGMAN, J.M., OREOPOULOS, D.G., VAS, S.I., Mod Pathol., 2, 1989, p. 69.

13.WENKER, J.C., BAKER, M.K., ELLIS, J.H., GLANT, M.D., AJR, 143, 1984, p. 573.

14.YATES, C.K., STREIGHT, R.A., Radiology 159, 1986, p. 83.

$\overline{\text { Manuscript received: } 11.02 .2019}$ 